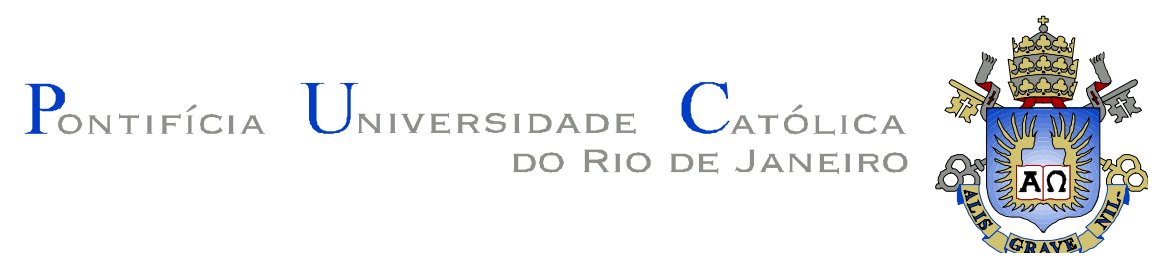

Patrícia Valéria Sathler de Queiroz

\title{
Mensuração do consumo de energia elétrica: algoritmo para detecção de potenciais usuários da termoacumulação como alternativa para deslocamento de carga
}

\section{Dissertação de Mestrado}

Dissertação apresentada como requisito parcial para obtenção do título de Mestre pelo Programa de PósGraduação em Metrologia da PUC-Rio. Área de Concentração: Metrologia para Qualidade e Inovação.

Orientador: Prof. Reinaldo Castro Souza Co-orientador: Prof. Maurício Nogueira Frota

Rio de Janeiro

Setembro de 2011 


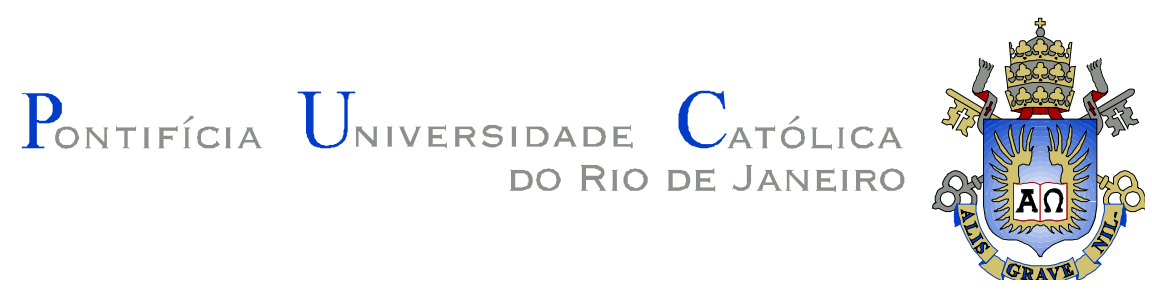

Patrícia Valéria Sathler de Queiroz

Mensuração do consumo de energia elétrica: algoritmo para detecção de potenciais usuários da termoacumulação como alternativa para deslocamento de carga

Prof. Reinaldo Castro Souza, PhD. Orientador

Programa de Pós-Graduação em Metrologia Pontifícia Universidade Católica do Rio de Janeiro - PUC-Rio

Prof. Maurício Nogueira Frota, PhD. Co-orientador Coordenador do Programa de Pós-Graduação em Metrologia Pontifícia Universidade Católica do Rio de Janeiro - PUC-Rio

Prof. João Carlos de Oliveira Aires, DSc. Universidade Gama Filho - UGF

Prof. José Francisco Pessanha, PhD. Universidade do Estado do Rio de Janeiro - UERJ

Prof. José Eugenio Leal Coordenador Setorial de Pós-Graduação do Centro Técnico Científico (PUC-Rio) 
Todos os direitos reservados. É proibida a reprodução total ou parcial do trabalho sem autorização da universidade, da autora e dos orientadores.

\section{Patrícia Valéria Sathler de Queiroz}

Graduou-se em Engenharia Elétrica na UGF (Universidade Gama Filho) em 2007. Pós Graduou-se em Engenharia de Segurança no Trabalho na UFRJ (Universidade Federal do Rio de Janeiro) em 2009. Colaborou no desenvolvimento no projeto de P\&D Light/Puc-Rio de "Racionalização do uso de energia e aplicação da termoacumulação como estratégia para proposição de tarifa diferenciada voltada a clientes comerciais de alto consumo", que motivou esta dissertação de mestrado.

Ficha Catalográfica

Queiroz, Patrícia Valéria Sathler de

Mensuração do consumo de energia elétrica : algoritmo para detecção de potenciais usuários da termoacumulação como alternativa para deslocamento de carga / Patrícia Valéria Sathler de Queiroz ; orientador: Reinaldo Casto Souza ; co-orientador: Maurício Nogueira Frota. - 2011.

98 f. : il. (color.) ; $30 \mathrm{~cm}$

Dissertação (mestrado)-Pontifícia Universidade Católica do Rio de Janeiro, Programa de Pós-Graduação em Metrologia para a Qualidade e Inovação, 2011.

Inclui bibliografia

1. Metrologia - Teses. 2. Consumo de Energia. 3. Eficiência Energética. 4. Termoacumulação. I. Souza, Reinaldo Castro. II. Frota, Maurício Nogueira. III. Pontifícia Universidade Católica do Rio de Janeiro. Programa de Pós-Graduação em Metrologia para a Qualidade e Inovação. IV. Título.

CDD: 389.1 
Este trabalho é dedicado à minha mãe Celina, que almejou o meu crescimento intelectual, ao meu marido William, que sempre me incentiva na busca do conhecimento. Ao meu orientador e amigo Reinaldo, que me deu a oportunidade de trilhar este novo caminho. Ao meu co-orientador Maurício, que abriu caminhos para esta dissertação. 


\section{Agradecimentos}

A Deus por me revigorar as energias, especialmente nos momentos mais difíceis e por ter colocado pessoas maravilhosas em meu caminho, verdadeiros anjos, que me apoiam e me fortalecem em todos os momentos.

Ao meu querido Guru, amigo e orientador professor PhD. Reinaldo Castro Souza, que com suas sábias colocações, me ajudaram nas melhores escolhas, enriquecendo o meu conhecimento.

Ao meu co-orientador professor PhD. Maurício Nogueira Frota, por seu entusiasmo e energia positiva, que me revitalizou em todo o percurso durante 0 mestrado e desenvolvimento da pesquisa de termoacumulação, também por suas valiosas sugestões e orientações.

Ao Sebastião G. de Oliveira, do Núcleo de Estatística Computacional (NEC) PUC-Rio, por ter realizado a programação do sistema gerador de curvas de cargas - SIMCAR, implementando os algoritmos, favorecendo ao bom andamento da pesquisa e do desenvolvimento deste trabalho.

A Fernanda Particelli e a equipe da Light do projeto de Termoacumulação, por ter disponibilizado todas as informações necessárias para o desenvolvimento do projeto.

A todos os professores do curso de Metrologia, que se empenharam em solidificar os conhecimentos básicos da área metrológica, focando na inovação e qualidade.

Aos meus amigos do mestrado, especialmente, Aguinaldo e Marcio, pela parceria e companheirismo durante o curso.

Aos funcionários da secretária do curso de Metrologia, pelas orientações a respeito das questões burocráticas a serem seguidas no curso.

Ao meu amigo e irmão Eric pelo apoio e sugestões técnicas e minha irmã Adriana, pelas revisões de texto. 


\section{Resumo}

Queiroz, Patrícia Valéria Sathler de Queiroz; Souza, Reinaldo Castro; Frota, Maurício Nogueira. Mensuração do consumo de energia elétrica: algoritmo para detecção de potenciais usuários da termoacumulação como alternativa para deslocamento de carga. Rio de Janeiro, 2011. 98p. Dissertação de Mestrado - Programa de Pós-Graduação em Metrologia (Área de Concentração: Metrologia para Qualidade e Inovação), Pontifícia Universidade Católica do Rio de Janeiro.

Esta dissertação desenvolveu-se de forma articulada com o projeto de P\&D LIGH-ANEEL, coordenado pelo Programa de Pós-Graduação em Metrologia (PósMQI), Projeto Ref. P\&D 003/2008, intitulado Racionalização do uso de energia e aplicação da termoacumulação como estratégia para proposição de tarifa diferenciada voltada a clientes comerciais de alto consumo. A dissertação teve como objetivo o desenvolvimento e validação de um algoritmo para identificação de clientes potenciais ao uso da tecnologia de termoacumulação. O trabalho orientou-se no contexto da crescente demanda por energia imposta ao setor elétrico pela expansão da economia, notadamente em horários de alta demanda. A motivação deste trabalho, atendendo aos interesses do PósMQI na área de energia, é oferecer uma ferramenta de auxílio à decisão para o planejamento energético das concessionárias. Como resultado obteve-se o mapeamento das curvas de cargas, obtidas por meio do sistema computacional desenvolvido (SIMCAR). Este sistema (amigável) permite enquadrar os clientes potenciais, segundo características de consumo estruturadas em três categorias indicativas do uso da termoacumulação: "muito potencial", "potencial" e "já modula". Dentre as conclusões, o algoritmo desenvolvido mostrou-se ferramenta eficaz de auxílio ao planejamento energético e a termoacumulação uma alternativa sustentável para adequação da matriz energética do país, podendo gerar economias de até $40 \%$.

\section{Palavras-chave}

$$
\text { Metrologia; Consumo de Energia; Eficiência Energética; }
$$

Termoacumulação. 


\section{Abstract}

Queiroz, Patrícia Valéria Sathler de Queiroz; Souza, Reinaldo Castro (Advisor); Frota, Maurício Nogueira (Co-Advisor). Measurement of electric energy consumption: algorithm for detecting potential users of thermo-accumulation as an alternative for load shift. Rio de Janeiro, 2011. 98p. MSc. Dissertation - Programa de Pós-Graduação em Metrologia (Área de Concentração: Metrologia para Qualidade e Inovação), Pontifícia Universidade Católica do Rio de Janeiro.

An algorithm to measure and detect potential thermo-accumulation customers among the clients of a distributing utility was proposed in this work. Such algorithm uses statistical methods for the treatment of "missing data" and is applied to the measured hourly demands of medium and high voltage customers of the utility. Basically, the thermo-accumulation technique can be defined as a strategy to shift load from peak hours to off-peak hours. The algorithm allows the construction of load curves from the observed load series after the removal of outliers and/or missing values. From the profile of such curves, the algorithm produces the classification of the clients into three possible categories: higly potential, just potential and non potential (i.e. an already modulated client). Also important to mention this dissertation, is part of an R\&D project developed for a brazilian southern utility. A friendly computer system named SIMCAR, developed in Visual Basic, implemented the proposed algorithm. As a final word, in surveys conducted with users of this technology, it was mentioned that the savings on energy consumption can be as high as $40 \%$.

\section{Keywords}

Metrology; Consumption of energy; Energetic Efficiency; Thermoaccumulation. 


\section{Sumário}

1 Introdução 13

1.1. Problematização e motivação 16

1.2. Hipótese 17

$\begin{array}{ll}\text { 1.3. Objetivo geral } & 17\end{array}$

$\begin{array}{ll}\text { 1.3.1. Objetivos específicos } & 17\end{array}$

$\begin{array}{ll}\text { 1.4. Metodologia } & 17\end{array}$

1.4.1. Classificação da pesquisa 18

1.4.2. Estrutura da dissertação 18

2 Eficiência energética no setor elétrico brasileiro 20

2.1. Principais ações brasileira de eficiência energética 22

2.1.1. Programa brasileiro de etiquetagem (PBE) 23

2.1.2. Programa nacional de conservação de energia elétrica (PROCEL) 24

2.1.3. O Programa CONPET 26

2.1.4. Programa de eficiência energética (PEE) 27

2.1.5. Lei de eficiência energética 28

2.2. Eficiência energética em edifícios 28

2.3. Características básicas do sistema elétrico brasileiro 29

2.3.1. Agentes institucionais $\quad 30$

2.3.2. Sistema de geração 31

2.3.3. Sistema de transmissão 32

2.3.4. Sistema de distribuição 33

2.3.5. Nível de tensão elétrica 33

2.3.6. Horário de ponta 34

2.4. Tarifa 34

2.4.1. Tipos de tarifas $\quad 35$

2.4.2. Grupos tarifários 36

3 Mapeamento das curvas de carga $\quad 38$

3.1. Curva de carga típica por natureza de consumidor 40

3.1.1. Curva de carga residencial 40

3.1.2. Curva de carga industrial 41

3.1.3. Curva de carga comercial 42 
3.2. Medição de energia elétrica 43

3.2.1. Medidores $\quad 44$

4 Termoacumulação: alternativa tecnológica para eficiência energética. 49

4.1. Fundamentos da termoacumulação 50

4.1.1. Formas de termoacumulação 51

4.1.2. Tecnologias de armazenamento para sistemas de termoacumulação 52

4.1.3. Tipos de sistemas de termoacumulação $\quad 54$

4.1.4. O funcionamento básico da termoacumulação 54

5 Algoritmo para detecção de clientes potenciais 56

5.1. Construção do algoritmo

5.1.1. Análise exploratória da população $\quad 57$

5.1.2. Levantamento da base de dados 58

5.1.3. Tratamento de dados aberrantes "outliers" 58

5.1.4. Tratamento de dados faltantes "missing data" 61

5.1.5. Descrição do algoritmo de detecção 65

5.1.6. Fluxograma básico do algoritmo de detecção 67

5.2. Sistema gerador de curvas de carga (SIMCAR) 68

5.2.1. Características do sistema 69

6 Testes e aplicações do algoritmo de detecção 71

6.1. Mapeamento das curvas de cargas $\quad 71$

6.2. Testes do algoritmo 72

6.3. Outras aplicações $\quad 74$

7 Conclusões e recomendações $\quad 76$

7.1. Produção acadêmica resultante da pesquisa de mestrado 77

8 Referências bibliográficas $\quad 78$

ANEXO I - Estudo do caso da EBVRJ 86

ANEXO II - Manual SIMCAR 92 


\section{Lista de tabelas}

Tabela 1 - Subgrupo Tarifário "A". 36

Tabela 2 - Subgrupo Tarifário "B". 36

Tabela 3 - Tabela de referência para enquadramento tarifário subgrupo A. $\quad 37$ 


\section{Lista de figuras}

Figura 1 - Evolução do consumo final por fonte $\quad 21$

Figura 2 - Etiqueta Nacional de Conservação de Energia 24

Figura 3 - Selo PROCEL 25

Figura 4 - Selo Conpet $\quad 27$

Figura 5 - Matriz de energia elétrica 32

Figura 6 - Tarifas praticadas no mundo $\quad 35$

Figura 7 - Distribuição do consumo final de energia elétrica 39

Figura 8 - Curva de carga típica do SIN, consumidor residencial 41

Figura 9 - Curva de carga típica do SIN - consumidor industrial 42

Figura 10 - Curva de Carga típica do SIN - consumidor comercial 42

Figura 11 - Medidor eletromecânico NANSEN M1A-T 45

Figura 12 - Medidor eletrônico NANSE $\quad 46$

Figura 13 - Instalação típica de reservatório de água gelada 53

Figura 14 - Instalação típica de um reservatório de gelo 53

Figura 15 - Instalações básicas de um sistema de termoacumulação 54

Figura 16 - Intervalo de confiança (96 medições de um dia útil) 60

Figura 17 - Intervalo de confiança (96 medições de um dia útil) com "outlier" 60

Figura 18 - Diagrama de blocos rotina tratamento de "outlier" 61

Figura 19 - Carga diária de um consumidor com "missings data" 63

Figura 20 - Tratamento "missing data" 64

Figura 21 - Fluxograma básico do algoritmo de detecção 68

Figura 22 - Tela principal do sistema computacional 69

Figura 23 - Gráfico dos resultados do mapeamento das curvas de carga 72

Figura 24 - Curva de carga cliente muito potencial 73

$\begin{array}{ll}\text { Figura } 25 \text { - Curva de carga cliente potencial } & 73\end{array}$

Figura 26 - Curva de carga cliente que já modula $\quad 74$

Figura 27 - Impacto da termoacumulação na curva de carga 75 


\section{Siglas e Abreviaturas}

ALIO - Asociación Latino-lberoamerica de Investigación Operativa

ANEEL - Agência Nacional de Energia Elétrica

ANP - Agência Nacional do Petróleo

AP - Audiência Pública

AT - Alta Tensão

BEN - Balanço Energético Nacional

$\mathrm{BIG}$ - Banco de Informações de Geração

BT - Baixa Tensão

CAG - Central de Água Gelada

CCEE - Câmara de Comercialização de Energia Elétrica

CCM - Centro de Controle de Medição

CITENEL - Congresso de Inovação Tecnológica em Energia Elétrica

CONPET - Programa Nacional da Racionalização do Uso dos Derivados do

Petróleo e d o Gás Natural

CMSE - Comitê de Monitoramento do Setor Elétrico

CNAE - Classificação Nacional de Atividades Econômica

CNPE - Conselho Nacional de Políticas Energética

DU - Dias Útil

EAT - Extra Alta Tensão

EBVRJ - Edifício da Bolsa de Valores do Rio de Janeiro

EPE - Empresa de Pesquisa Energética

ERPO - Encontro Regional de Pesquisa Operacional

GLD - Gerenciamento pelo Lado da Demanda

IFORS - The International Federation of Operational Research Societies

INMETRO - Instituto Nacional de Metrologia, Qualidade e Tecnologia

MME - Ministério de Minas e Energia

MT - Média Tensão

OIML - Organização Internacional de Metrologia Legal

ONS - Operador Nacional do Sistema Elétrico

PBE - Programa Brasileiro de Etiquetagem

$\mathrm{PCH}$ - Pequena Central Hidroelétrica

P\&D - Pesquisa e Desenvolvimento

PDE - Plano Decenal de Expansão de Energia

PEE - Programa de Eficiência Energética

PEN - Plano Nacional de Operação Energética

PPH - Pesquisa de Posses e Hábitos

PROCEL - Programa Nacional de Conservação de Energia Elétrica

SIMCAR - Sistema Gerador de Curva de Carga

SIN - Sistema Interligado Nacional

SOBRAPO - Sociedade Brasileira de Pesquisa Operacional

UAT - Ultra Alta Tensão 